Revue d'histoire de l'Amérique française

REYUE D.HISTOIRE DE L'AMÉRIQUE FRANÇAISE

\title{
BERCUSON, David J., Kerry M. ABEL, Donald H. AKENSON, Peter A. BASKERVILLE, John M. BUMSTED et John G. REID, COLONIES: Canada to 1867. Toronto, McGraw-Hill Ryerson Limited, 1992. x-538 p.
}

\section{Pierre Tousignant}

Volume 47, numéro 1, été 1993

URI : https://id.erudit.org/iderudit/305184ar

DOI : https://doi.org/10.7202/305184ar

Aller au sommaire du numéro

Éditeur(s)

Institut d'histoire de l'Amérique française

ISSN

0035-2357 (imprimé)

1492-1383 (numérique)

Découvrir la revue

Citer ce compte rendu

Tousignant, P. (1993). Compte rendu de [BERCUSON, David J., Kerry M. ABEL, Donald H. AKENSON, Peter A. BASKERVILLE, John M. BUMSTED et John G. REID, COLONIES: Canada to 1867. Toronto, McGraw-Hill Ryerson Limited, 1992. x-538 p.] Revue d'histoire de l'Amérique française, 47(1), 93-96.

https://doi.org/10.7202/305184ar d'utilisation que vous pouvez consulter en ligne. 


\section{COMPTES RENDUS}

BERCUSON, David J., Kerry M. ABEL, Donald H. AKENSON, Peter A. BASKERVILLE, John M. BUMSTED et John G. REID, COLONIES: Canada to 1867. Toronto, McGraw-Hill Ryerson Limited, 1992. $\mathrm{x}-538 \mathrm{p}$.

Ce nouveau manuel d'histoire du Canada publié l'année du cent vingtcinquième anniversaire de la Fédération canadienne de 1867 confirme une fois de plus que la déesse Clio est bien fille de son temps et qu'elle doit conséquemment répondre aux différentes préoccupations et perspectives historiques de chaque époque.

Il y a un quart de siècle, lors du centenaire de la «Confédération», l'on croyait en la possibilité de produire un manuel unique destiné à favoriser l'unification de la «nation canadienne» a mari usque ad mare. C'est ainsi qu'en 1968, la maison d'édition Holt, Rinehart et Winston lançait, en versions anglaise et française, sous les titres Canada - Unity in Diversity/ Canada - Unité et Diversité, un ouvrage conçu, comme le précise l'avantpropos de Marcel Trudel, dans le but de «réunir tout ce qu'un Canadien [d'où qu'il soit] doit savoir aujourd'hui du passé de son pays» (un compte rendu détaillé de cet ouvrage est paru dans la RHAF, 22,3 (décembre 1968): 450466). La rédaction en fut confiée à une «équipe mixte» comprenant trois historiens francophones diplômés de l'Université Laval (Jean Hamelin, Fernand Ouellet et Marcel Trudel) et un historien anglophone, Paul G. Cornell, formé à l'Université de Toronto.

Depuis environ 25 ans, les préoccupations et les perspectives historiques ont manifestement changé. Si, dans les années 1960, à l'époque de la Commission royale d'enquête sur le bilinguisme et le biculturalisme, l'on considérait généralement la fédération canadienne en termes de dualité ethnoculturelle, aujourd'hui c'est la double dimension de la pluriethnicité et du multiculturalisme qui polarise l'attention. COLONIES: Canada to 1867 illustre bien ces nouvelles perceptions de la réalité canadienne. Le professeur David Jay Bercuson était l'homme tout désigné pour prendre l'initiative de la réalisation de ce manuel. Né à Montréal, il y reçut une partie de sa formation académique qu'il compléta à Toronto avant de faire carrière à l'Université de Calgary.

Dans la préface et l'introduction, le maître d'œuvre précise que le plan 
de l'ouvrage a été élaboré en fonction d'un large cadre d'histoire coloniale s'étendant de Terre-Neuve à l'île de Vancouver afin de bien montrer et démontrer l'ampleur des «différences régionales» qui ont caractérisé l'histoire du Canada depuis les origines jusqu'à nos jours. Le régionalisme est présenté comme une donnée historique fondamentale pour comprendre que «the amount of diversity was mirrored in the extent of disunity [1a grandeur de la diversité se reflétait dans l'étendue de la désunion]» (p. 5). Les facteurs qui contribuèrent à façonner ces «différences régionales» furent aussi nombreux que variés, dont, entre autres, la géographie, le climat, les activités économiques, les divisions et les rivalités ethnoculturelles. "At Confederation, Canada was a desunited, diverse, physically separated, and very complex society.» (p. 3)

Selon David J. Bercuson, il faut tout ignorer de l'histoire coloniale pour prétendre que la création du Dominion du Canada fut l'œuvre de «deux peuples fondateurs». Il s'agit là d'un mythe sans fondement historique puisque l'existence même de collectivités anglophone et francophone séparées empêcha la transformation en une nation d'une «collection de colonies disparates, dispersées à travers le continent». Aussi peut-il affirmer, en conclusion de l'ouvrage, que si les Pères de la Confédération ont réalisé «la plupart des objectifs limités qu'ils s'étaient fixés», par contre, l'union fédérale de 1867 ne créa assurément pas de nation canadienne (p. 521-522).

La rédaction des onze chapitres a été confiée à cinq historiens anglophones œuvrant dans des champs de spécialisation diversifiés et dont les ports d'attache universitaires se situent d'Halifax à Victoria en passant par Ottawa, Kingston et Winnipeg. Il n'est pas fait mention de la division des tâches ni dans la table des matières ni dans la présentation du maître d'œuvre. Il m'a donc fallu remonter à la source — c'est-à-dire à l'éditeur lui-même pour savoir «qui avait écrit quoi». Les deux premiers chapitres, qui couvrent la période coloniale de Nouvelle-France, et le troisième, consacré à la traite des fourruresdu XVI ${ }^{\mathrm{e}}$ siècle à la monopolisation par la Hudson's Bay Company, ont été rédigés par Kerry $\mathrm{M}$. Abel; les quatrième et cinquième, qui portent sur les débuts du régime britannique au Canada, sont de la plume de John M. Bumsted; les trois suivants (VI, VII et VIII), qui traitent du développement et des transformations socio-économiques du British North America au $\mathrm{XIX}^{\mathrm{e}}$ siècle, ont été composés par Peter A. Baskerville; les neuvième et dixième, respectivement intitulés «The Pre-Confederation Roots of Canadian Regionalism» et «The Confederation Question, 1864-1867», ont été confiés à John G. Reid; enfin, l'auteur du onzième et dernier chapitre, Donald H. Akenson, y traite de l'influence déterminante de la société et de la civilisation européennes.

Du point de vue pédagogique, COLONIES: Canada to 1867 ne saurait rivaliser avec ORIGINS. Canadian History to Confederation (Toronto, Holt, Rinehart and Winston of Canada Ltd, 1992, $2^{\mathrm{e}}$ édition) de R. Douglas Francis, Richard Jones et Donald B. Smith (voir le compte rendu de Pierre Trépanier, dans la $R H A F, 42,4$ (printemps 1989): 626-629). Faute de table des matières détaillée (on n'y trouve qu'une énumération sommaire des intitulés des onze chapitres), il faut préalablement se familiariser avec le contenu pour 
parvenir à repérer l'information désirée. Le plaisir de la découverte ne saurait remplacer les inconvénients didactiques de la recherche à l'aveuglette quand il s'agit d'un ouvrage destiné à l'enseignement. On peut constater que ce manuel n'a pas été planifié en fonction d'objectifs pédagogiques bien définis. Lorsqu'on se donne la peine de dresser la liste des sous-titres de chacun des chapitres, il en ressort clairement un manque d'unité et de cohérence dans la façon d'aborder et de traiter les questions et les problèmes.

La différenciation entre les auteurs est même si notable qu'elle rend les comparaisons inévitables. Les meilleures contributions et les plus dignes de mention me paraissent être celles de Kerry M. Abel et de Peter A. Baskerville. Non seulement ces auteurs offrent-ils une couverture équilibrée de la matière traitée dans chacun de leurs chapitres, mais ils s'efforcent de rendre compte de l'historiographie la plus récente.

Étant donné son domaine de spécialisation, Kerry M. Abel peut traiter à son aise des peuples autochtones et de leurs relations avec les colonisateurs européens, notamment avec les colons français de Nouvelle-France. La conclusion de son deuxième chapitre vaut d'être partiellement citée: "The French immigrants brought with them ideas and institutions from a homeland in transition between the ancien régime and modernity... Unable and perhaps unwilling to reproduce in the New World their lives from the old, they evolved a society that represented something uniquely in between.» (p. 76)

Il est regrettable que l'histoire des débuts du régime britannique (17631791), confiée à John M. Bumsted, ait été mal planifiée et mal traitée au point de nécessiter une révision du chapitre IV dans l'éventualité d'une seconde édition. L'auteur se permet des affirmations si fantaisistes qu'elles ne peuvent que paraître grotesques à mes yeux de spécialiste de la période. En voici quelques exemples: "The inability of the Quebec government to eliminate the seigneurial system [à la veille de la révolution américaine] further detracted from the attraction of that province for restless Americans... Quebec was spared a wholesale land grab only by the complications of the seigneurial system in the territory under its control.» (p. 123)

Fort heureusement, Peter A. Baskerville prend son rôle d'historien plus au sérieux et sa contribution est exemplaire tant par la clarté de l'exposé que par la richesse du contenu. On ne saurait trop louer l'approche méthodologique qu'il utilise dans ses trois substantiels chapitres qui, à eux seuls, représentent le tiers de l'ouvrage. Il prend toujours soin de préciser les questions et de définir les problèmes par rapport à l'historiographie avant de fournir des éléments de réponse qu'il s'efforce de nuancer tout au long de son exposé. Il vaut d'illustrer par un exemple sa démarche professionnelle d'historien avisé.

Dans le chapitre VII intitulé «The Business Cultures of Pre-Confederation Canada», Peter A. Baskerville soulève la grande question des conséquences socio-économiques de la Conquête. Après s'être demandé si, du fait du changement de métropole, l'on peut soutenir la thèse d'un «nouvel ordre économique», il passe en revue les principales interprétations à ce sujet et il résume ainsi son propre point de vue: 
Existing social structures were able to accommodate and shape capitalist development rather than be reshaped by such economic change. This is an important (although far from unchallenged) perspective. It forces us, as students, to be cautious about attributing great consequences to presumed watershed events like conquests and revolutions. This is not to imply that nothing ever changes: rather dramatic events rarely sweep all before them. What the Conquest transformed in terms of North America's economy had more to do with ethnicity and direction of trade than with business practice and structure. (p. 286)

Si tout le contenu de ce manuel avait été ainsi pétri de science historique, il m'aurait été agréable d'en recommander chaleureusement l'adoption au niveau du premier cycle universitaire. 